Gastroenterologia. 1939;64:I-IV

\title{
Contents, Vol. 64, 1939
}

Vorwort - Avant-propos - Introduction

Bacon, H. E. and W. A. H. Sche††íer, Melanosis Proctocoli. 68

Baumgärtel, 7'., Biologische Grundlagen der individuellen Coli-Implantations-Therapie zur Normalisierung der bakteriellen Dickdarmflora 304

Brocher, J. E. W., Demonstration de documents radiologiques co $\pi$ cernant trois cas rares du tube digestif 274

Demote, M. et /. Neeser, L'Hyperazotémie dans les hémorragies digistives 208

- et G. Henny, Ulcère bénin de la grande courbure d'aspect néoplasique 282

Dobre $\uparrow$ f, M. und /. Kaltscheff, Ein Beitrag über die Magensaftsekretion nach Typhus abdominalis 44

Ecke, W. und O. Simon, Schnelle Rückbildung großer Ulkusnischen unter diätetischer Behandlung1

Eínhorn, M., Über den Wert der Fadenprobe zur Diagnose von peptischen Geschwüren 65

Groß, C, Un carcinome pylorique d'aspect radiologique trompeur: image d'ulcère duodenal 286

Grott, J. W., Über die Diagnose der latent verlaufenden chronischen

Bauchspeicheldrüsenentzündung 73

Gutmann, R. A., Ulères bénins, ulcères transformés et cancers ulcériformes 259

Henny, G., vide Demote, M. Kaltscheff, J., vide Dobreff, M.

Kapp, H., Ulcus duodeni als Unfallserkrankung 290

Lüdin, M., Das Röntgenbild des Dünndarmes bei der einheimischen Sprue 191

Lyon, E;, The Position of Hepatopathy within the Etiology of Ostei tis Deformans Paget 84

Mahlo, Zur Genese der perniziösen Anämie 19

Markert, W., Der Einfluß von Pankreastumoren auf die Leistungsfähigkeit der Niere $\quad 324$

Michaud, M., Demonstrations cliniques d'une série d'affections de Гoesophage 263

Neeser, J., vide Demote, M.

Oltramare, J. H., Reflexions sur $\Gamma$ ulcère péptique .... 267 Paschoud, H., Les raisons de $\Gamma$ emploi des ondes electromagnétiques en chirurgie opératoire gastro-entérologique 226

Piotet, G., Nouvelles remarques sur les invaginations 
Sche $\uparrow$ †ler, W. A. H., vide Bacon, H. E.

Sílva-Melío, Über die Yatrenbehandlung bei Darmkrankheiten . 93

Simon, O, vide Ecke, W.

Sparchez, T. und E. Viciu, Takata-Ara-Reaktion und ihr klinischer

Wert in der Diagnose der Lebererkrankungen .... 23 Stalder, H., Zur Kritik der internen

Ulkustherapie . . . 198 Steinítz, ii., Untersuchungen zur Pathologie des Fruktose-Stoffwechsels. Reine Fruktosurie bei Qeschwistern. Diabetes und

Fruktose-Stoffwechsel $\quad 334$

Tecon, R. M., A propos d'un cas de sprue 177

Viciu, E;, vide Sparchez, T.

Vogel, E., Ein Okklusionsblatt für das Rektoskop .... 91

Zabel, E., Über Balantidienkolitis 12

Übersichtsreferate - Reviews - Revues

Kalíós, P., Über nutritive Allergie 234

Simon, K· Th., Kurze Übersicht über die Beziehungen zwischen den

verschiedenen Magenaffektionen und den Veränderungen der

Blutzusammensetzung 348

Gesamtliteratur des In- und Auslandes .... 52, 145, 249, 374 Buchbesprechungen - Book-Notices

- Livres Nouveaux 58, 168, 257, 381 